\title{
CPT-pump-probe measurement of the Cs clock transition DC Stark shift
}

\author{
Jean-Luc Robyr, Paul Knowles, and Antoine Weis \\ Department of Physics \\ University of Fribourg, CH-1700 Fribourg \\ Email: jean-luc.robyr@unifr.ch
}

\begin{abstract}
We report progress in measuring the third order electric polarizability of the Cs ground states using a Ramsey pump-probe experiment on coherent population trapped (CPT) atoms in a thermal atomic beam. We give a short description of the apparatus as well as the Fourier transform method used to monitor the phase and frequency of the Ramsey signal. Analysis of a typical data set is shown, proving the consistency of the method. We show that the motional magnetic field phase shift can be used to test the reliability of the electric field modeling. Finally, we give a preliminary value for the Cs ground state polarizability and compare it to previous published values of the DC Stark shift.
\end{abstract}

\section{INTRODUCTION}

The second is defined by the unperturbed ground-state hyperfine frequency splitting in ${ }^{133} \mathrm{Cs}$ (ISO 31-1). Realizing the second requires control and understanding of the systematic effects [1], [2] that perturb that transition. One effect is the AC Stark shift arising from the blackbody radiation spectrum of the finite temperature clock environment. In practice, the blackbody radiation shift can be calculated from the Cs ground state third-order polarizability $\alpha_{0}^{(3)}(F)$ which we measure in a CPT-based Ramsey pump-probe experiment similar to [3] and [4] on a Cs thermal atomic beam. The polarizability induces a shift in the resulting Ramsey fringe as a function of the DC electric field applied in the free evolution region of the atom's flight.

The energy shift produced by a DC electric field $\mathbb{E}$ is described using the electric polarizability $\alpha$ as $\Delta E\left(n, L_{J}, F, m_{F}\right)=-\frac{1}{2} \alpha \mathbb{E}^{2}$. The constant $\alpha$ is traditionally expanded in power series, both in multipole order (subscript) and perturbation order (superscript). Neglecting the (small) quadrupole hyperfine interaction, the lowest order terms (i.e., third) affecting the $\Delta m_{F}=0$ ground-state Cs hyperfine transitions, produce a frequency shift $\delta \nu=-\frac{1}{2 h} \alpha \mathbb{E}^{2}$ [5], [6] with

$$
\alpha=\frac{16}{7} \alpha_{0}^{(3)}(F=4)+\alpha_{2}^{(3)}(F=4) \frac{3 m_{F}^{2}-16}{28} f(\theta),
$$

where $\alpha_{0}^{(3)}$ and $\alpha_{2}^{(3)}$ are the third order scalar and tensor polarizabilities, $m_{F}$ is the magnetic quantum number defined by the quantization axis (magnetic field), and $f(\theta)=$ $3 \cos ^{2}(\theta)-1$ is a function of the relative angle between $\hat{\mathbb{E}}$ and the quantization axis. Note that $f(\theta)$ averages to zero for isotropic blackbody radiation, and that the Ramsey method makes the shift sensitive to the effective average squared field,
$\left\langle\mathbb{E}^{2}\right\rangle$. For a $20 \mathrm{kV} / \mathrm{cm}$ static electric field, the expected scalar shift in Cs is $830 \mathrm{~Hz}$, and the tensor shift is about $3 \mathrm{~Hz}$.

\section{A. Motional magnetic field}

Atoms flying through the electric field $\overrightarrow{\mathbb{E}}$ experience an additional magnetic field $\vec{B}_{\text {mot }}$ given by

$$
\vec{B}_{m o t}=-\frac{\vec{v}}{c^{2}} \times \overrightarrow{\mathbb{E}}
$$

where $\vec{v}$ is the velocity of the atom. This motional field produces a Zeeman shift of the $\Delta m_{F}=0$ hyperfine transitions but due to its linear velocity dependence this shift results in a phase change

$$
\Delta \phi=\left(g_{F=4}-g_{F=3}\right) m_{F} \mu_{B} \frac{\langle\mathbb{E}\rangle}{\hbar} \frac{L}{c^{2}}
$$

of the Ramsey resonance, where $g_{i}$ is the Landé factor of level $i, m_{F}$ is the magnetic quantum number of both ground states (our experiment presently probes phase shifts of levels with identical $\left.m_{F}\right),\langle\mathbb{E}\rangle$ is the mean electric field seen by the atoms between the pump and the probe zones, separated by $L$. This phase shift produces a small displacement of the Ramsey resonance center frequency and thus mimics an atomic frequency shift. However, from this measured phase, the mean electric field value can be inferred and then compared to an electric field model prediction to give confidence in the latter.

\section{B. Extracting frequency and phase}

The Ramsey resonance lineshape $S\left(\omega_{R F}\right)$ can be expressed as the integral of the phase accumulated by each velocity class during the interaction with the electric and magnetic fields weighted by the atomic velocity distribution, $\rho(v)$. The accumulated phase is given by a term oscillating at the difference between the microwave $\omega_{R F}$ and atomic $\omega_{a t}$ frequencies and includes a constant offset $\phi$.

$$
S\left(\omega_{R F}\right)=\int_{0}^{\infty} \rho(v) \cos \left[\left(\omega_{R F}-\omega_{a t}\right) \frac{L}{v}+\phi\right] d v .
$$

Taking the Fourier transform of $S\left(\omega_{R F}\right)$ yields

$$
\mathcal{F}\left[S\left(\omega_{R F}\right)\right] \propto \rho(T) \exp \left[i\left(\omega_{a t} T+\phi\right)\right],
$$

where $\rho(T)$ is the atomic time-of-flight distribution. The argument of the complex exponential contains information on both atomic frequency $\omega_{a t}$ and phase $\phi$ of the Ramsey signal $S\left(\omega_{R F}\right)$ permitting their clear separation and extraction. The 


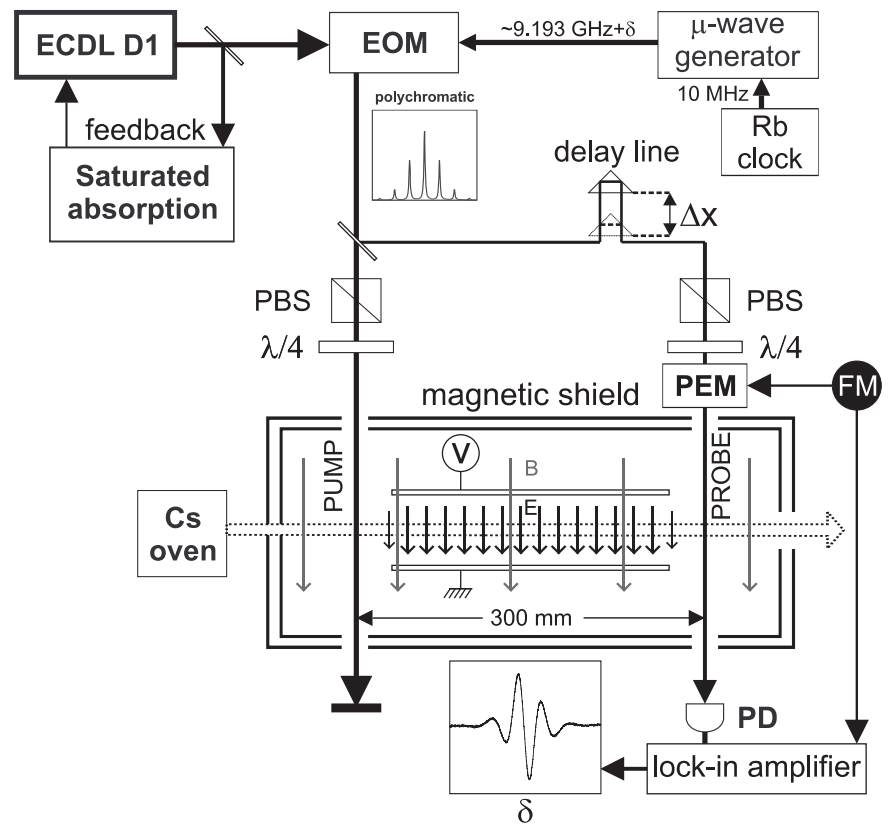

Fig. 1. Experimental setup for the measurement of the Stark shift. In order to produce a phase coherent bichromatic light field, a Cs $D_{1}$ extended cavity diode laser beam is phase modulated at the Cs hyperfine frequency using a electro-optic modulator (EOM). The modulated laser beam is split into a strong pump and a weak probe and sent to a Cs atomic beam experiment to perform Ramsey interrogation. The probe-beam transmitted intensity is recorded while scanning the microwave frequency. The DC Stark shift is obtained applying an electric field to the atoms during their flight between the pump and the probe zones.

Fourier transform is therefore a powerful tool to distinguish between Stark frequency shift and motional phase shift: similar techniques have been used in [7], [8].

\section{EXPERIMENTAL SETUP}

The main elements of the present experimental scheme have already been described in detail in [9] and here we will focus on the new elements. Figure 1 shows the actual experimental configuration. A Cs $D_{1}$ extended cavity diode laser is stabilized to a chosen Cs hyperfine transition (normally the $F=3 \longrightarrow F^{\prime}=3$ ) using saturated absorption in an atomic vapor cell. The light is sent through a commercial lithium niobate electro-optic modulator (EOM), which accepts a microwave signal (whose frequency can be scanned around the Cs ground state hyperfine frequency $\sim 9.2 \mathrm{GHz}$ ), and produces a frequency modulated spectrum, containing multiple sidebands all separated by the modulation frequency. The resulting spectrum generates coherent population trapping (in this case, the carrier and the $-\omega_{R F}$ bands are used). The modulated beam is split into a strong pump beam and a weak probe beam. The pump and probe beams pass through polarization optics to create the desired circular polarization before being sent to the thermal Cs atomic beam. The interaction between the light and the atoms occurs in a magnetically shielded environment with a $3.6 \mu \mathrm{T}$ DC magnetic field. The atomic beam first interacts with the pump laser beam creating a coherence between the Cs hyperfine ground states which then evolves

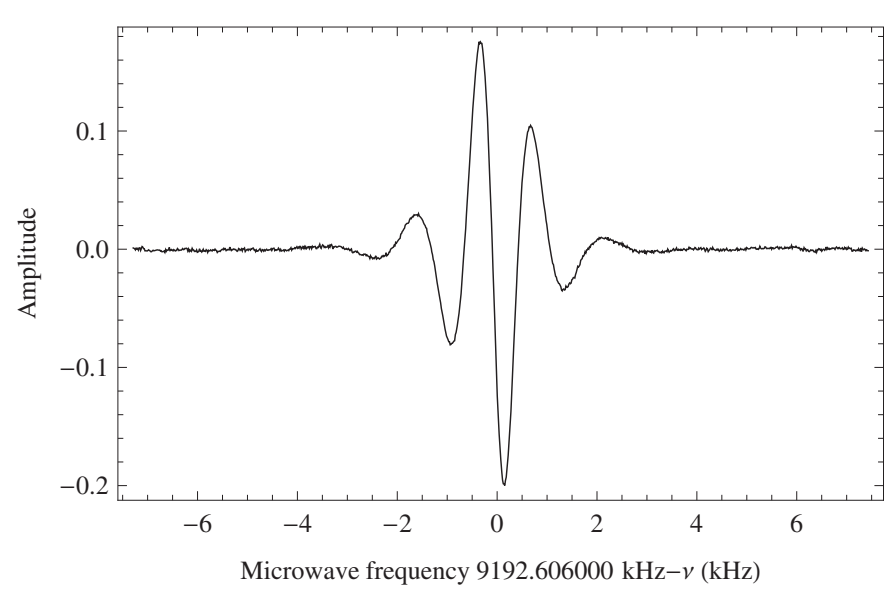

Fig. 2. Averaged Ramsey fringe pattern (15 scans) of the Cs hyperfine transition $m_{F}=+1 \rightarrow m_{F}=+1$, with a central fringe period of $\sim 1 \mathrm{kHz}$. The dispersive lineshape is due to the difference of microwave phases between the pump and probe zones and can be controlled by the optical delay line.

over a $300 \mathrm{~mm}$ path length during which it passes between two $260 \mathrm{~mm}$ long electrodes with given potential difference. The electric field between the plates shifts the atomic coherence oscillation frequency due to the Stark perturbation. Finally the phase of the coherence is probed by recording the transmitted laser intensity of the probe beam in the second interaction zone while scanning the microwave frequency, thus producing typical Ramsey spectra (Fig. 2).

\section{A. Electric field}

The electric field is produced by applying a known voltage to one of the two conductive rectangular float glass plates, while the other is grounded. The plate separation is defined by $6.065 \mathrm{~mm}$ thick optical flats. Two grounded metal plates, with apertures collimating the $\mathrm{Cs}$ beam, are placed at the entrance and exit of the capacitor to shield the light interaction zones from electric fringe fields. The applied high voltage is measured by a calibrated digital voltmeter (with resolution of $\left.10^{-4}\right)$, and remains stable to better than that resolution for the typical measurement time of 10 minutes. A technical problem prevented the use of negative voltages exceeding $-2000 \mathrm{~V}$. Finite element electric field modeling is used in the data analysis to extract the polarizability [10].

\section{EXPERIMENTAL RESULTS}

\section{A. Ramsey fringe data}

Ramsey fringe spectra are recorded for each of the seven $\Delta m_{F}=0$ hyperfine transitions, split by the $3.6 \mu \mathrm{T}$ field. Due to the $\sim 1 \mathrm{~ms}$ precession time in the fields, the period of the central fringe is about $1 \mathrm{kHz}$. Each resonance spectrum is obtained ramping the microwave frequency over $\pm 7.5 \mathrm{kHz}$ around the center of the fringe. The background is removed using a quadratic fit function. The phase of the Ramsey resonance can be accurately controlled by an optical delay line. Figure 2 shows a typical Ramsey fringe spectrum for the $m_{F}=+1 \rightarrow m_{F}=+1$ hyperfine ground state transition. 


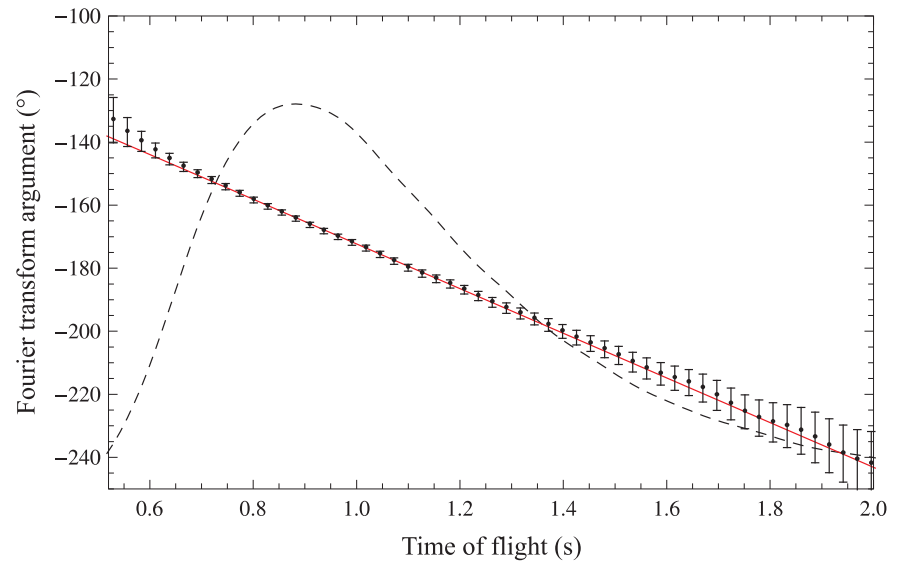

Fig. 3. Fourier transform data of recorded Ramsey signal showing the linear dependence of the argument $\omega_{a t} T+\phi$ in Eq. (4) in function of the time of flight. Each data point is weighted proportionally to the time of flight distribution in the beam (dashed line). A weighted linear fit is used to extract both the atomic frequency $\omega_{a t}$ and the Ramsey fringe phase $\phi$.

\section{B. Fourier transform of Ramsey data}

A discrete Fourier transform is applied to the Ramsey spectra. To avoid noise coming from asymmetric ends of the spectrum, a Tukey windowing function is applied to the data before transformation. The transformed data become functions of the atomic time-of-flight with a minimal time interval between two data points determined by the microwave scan span. However, since the applied frequency window goes to zero and there is no signal beyond the span limits, the effective span can be arbitrarily extended during the Fourier transform. As consequence, we are not limited in the time resolution of the Fourier transform. Figure 3 shows typical data from the Fourier transform's argument $\omega_{a t} T+\phi$ in Eq. (4). Each data point is attributed a weight proportional to the corresponding signal intensity from the time-of-flight distribution $\rho(T)$ (dashed line in Fig. 3). The atomic frequency $\omega_{a t}$ as well as the Ramsey fringe phase $\phi$ are extracted by a weighted fit of a straight line.

\section{Motional magnetic field}

The motional magnetic field produces a phase change of the Ramsey resonance as a function of the applied electric field, as described above. The Fourier transform analysis of the Ramsey spectra for different applied voltages gives access to this phase shift. Figure 4 shows the motional phase shift of the six $\Delta m_{F}=0$ hyperfine transitions with $m_{F}= \pm 1, \pm 2, \pm 3$ as fit with a linear function. As expected from Eq. (2) the slope depends linearly on $m_{F}$. The linear fits therefore give measurements of $\langle\mathbb{E}\rangle$ which can be compared to the mean electric field value obtained from the field modeling, useful to verify the accuracy of the latter. The quantity compared is the effective electrode separation $d_{\text {eff }}$ defined as

$$
\langle\mathbb{E}\rangle=\frac{V}{d_{\mathrm{eff}}},
$$

where $\langle\mathbb{E}\rangle$ is the mean electric field between the pump and probe interaction and $V$ the applied voltage. Table I shows

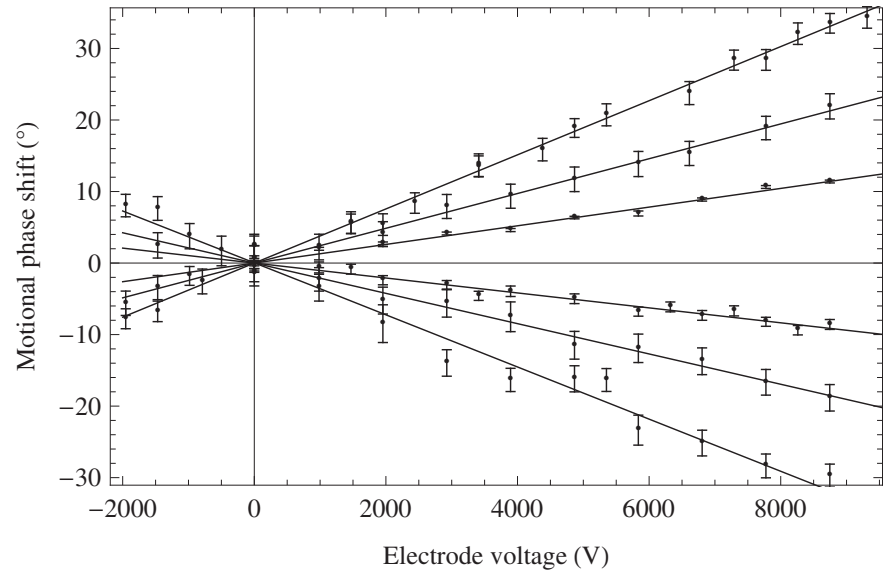

Fig. 4. Measurement of the Ramsey resonance phase as function of the applied electrode voltage for hyperfine transitions with $\Delta m_{F}=0$ and $m_{F}= \pm$ $1, \pm 2, \pm 3$. The linear fit of these data gives access to the mean electric field experienced by the atoms on their flight between the pump and probe interactions.

\begin{tabular}{lll}
\hline & Field modeling & Measurement \\
$d_{\mathrm{eff}}$ & $5.90(2) \mathrm{mm}$ & $5.83(8) \mathrm{mm}$ \\
\hline
\end{tabular}

TABLE I

VALUES FOR THE EFFECTIVE DISTANCE BETWEEN THE ELECTRODE PLATES OBTAINED WITH THE ELECTRIC FIELD NUMERICAL MODELING AND FROM THE MOTIONAL MAGNETIC FIELD PHASE SHIFT MEASUREMENT.

that the model and measurements agree. We currently work on improving the statistical and systematic uncertainties of the measurement.

\section{Stark shift}

The Cs ground state Stark shift is obtained by measuring the change of atomic frequency $\omega_{a t}$ as a function of the applied electrode voltage using the method described above. Figure 5 shows the Stark frequency shift for the $m_{F}=+1 \rightarrow m_{F}=+1$ Cs ground state hyperfine transition. A second order polynomial is fit to the data for extracting the quadratic coefficient, itself proportional to the electric polarizability of the Cs ground states. The linear coefficient is consistent with zero, as expected. The voltage is converted into the effective mean of the squared electric field $\left\langle\mathbb{E}^{2}\right\rangle$ seen by the atoms between the pump and probe interactions using numerical modeling of the electric field. A value of the total electric polarizability for each of the seven Cs ground states hyperfine transition is obtained and the polarizability difference of any pair of grounds states can be extracted using Eq. (1). Preliminary data with fit are shown in Fig. 6. The relative orientation of the electric and magnetic field for these measurement is $\theta=85(1)^{\circ}$. A preliminary value for $\alpha_{0}^{(3)}$ has been extracted from the fit and is compared, in Table II, with the two latest DC Stark shift measurements (which between themselves showed a discrepancy of more than $5 \sigma$ ). 


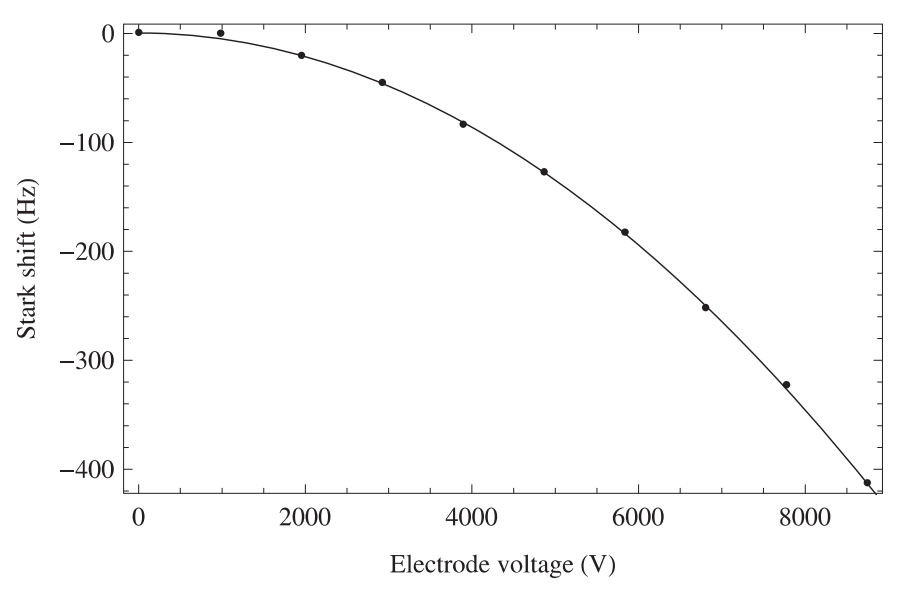

Fig. 5. Cs ground state Stark frequency shift of the $m_{F}=+1 \rightarrow m_{F}=+1$ hyperfine transition. Statistical uncertainties on the points are smaller than the point size. The continuous line is a second degree polynomial fit.

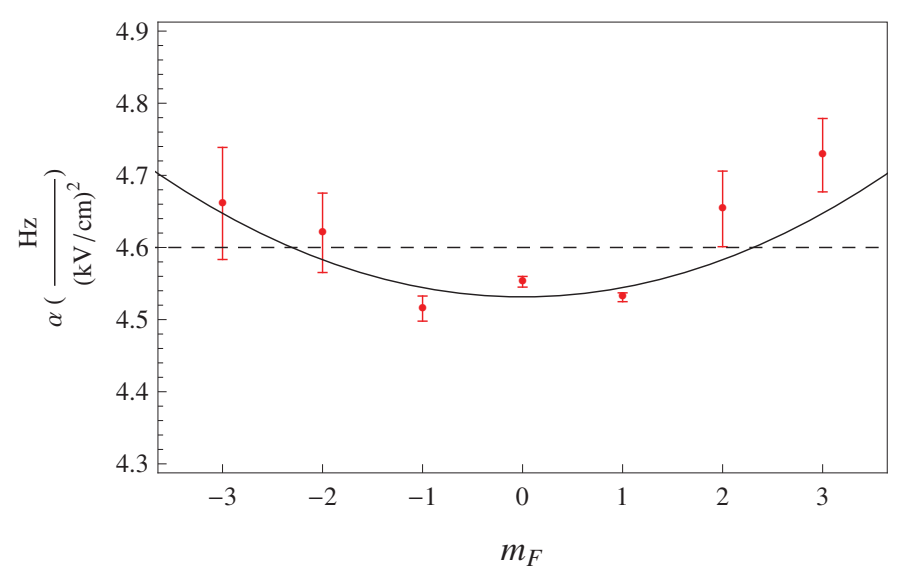

Fig. 6. Cs ground state electric polarizability extracted for each of the seven hyperfine transitions $\left(4, m_{F} \leftrightarrow 3, m_{F}\right)$. Error bars come from the measurement statistics with sizes depending on the signal to noise ratio of the Ramsey fringe, which is higher for low $m_{F}$ transitions. The continuous line shows the fit with Eq. (1). The dashed line shows only the scalar contribution in $\alpha$ from Eq. (1).

\section{CONCLUSION}

The Fourier analysis of the Ramsey spectra phase and center frequency has been successfully applied to measured data, extracting the Stark frequency shift, and the motional phase needed to test the electric field model which itself permits the third order Cs ground state polarizability $\alpha_{0}^{(3)}$ to be inferred. Our preliminary value for $\alpha_{0}^{(3)}$ was compared to previous measurements of the DC Stark shift. Further work will focus on improving the statistical uncertainties of the measurements and characterize and reduce the systematic effects.

\section{ACKNOWLEDGMENT}

The authors thank the mechanical and electronics workshops of the Fribourg University Physics Department for their skillful support, and the Swiss National Science Foundation (SNF \#200021-117841) for funding this project. Thanks go to Z. Andjelic, of ABB Corporate Research, Baden, for the field modeling.

\begin{tabular}{lll}
\multicolumn{4}{c}{ Measurement of $\alpha_{0}^{(3)}\left(\frac{\mathrm{Hz}}{(\mathrm{kV} / \mathrm{cm})^{2}}\right)$} \\
\hline Rosenbusch et al. & $1.997(4)$ & [11], [12] \\
Godone et al. & $1.78(4)$ & [13] \\
this work (preliminary) & $2.01(2)$ & \\
\hline
\end{tabular}

TABLE II

COMPARISON OF OUR PRELIMINARY MEASUREMENT OF THE THIRD ORDER ELECTRIC POLARIZABILITY AND TWO PREVIOUS MEASUREMENTS OF THE DC STARK SHIFT IN THE CS GROUND STATES, WHICH, BETWEEN THEM, SHOW A $>5 \sigma$ DISCREPANCY.

\section{REFERENCES}

[1] R. Wynands and S. Weyers, "Atomic fountain clocks," Metrologia, vol. 42, pp. S64-S79, 2005. [Online]. Available: http://www.iop.org/EJ/ abstract/0026-1394/42/3/S08

[2] J. H. Shirley, W. D. Lee, and R. E. Drullinger, "Accuracy evaluation of the primary frequency standard NIST-7," Metrologia, vol. 38, no. 5, pp. 427-458, 2001. [Online]. Available: http: //stacks.iop.org/0026-1394/38/427

[3] J. E. Thomas, P. R. Hemmer, S. Ezekiel, C. C. Leiby, R. H. Picard, and C. R. Willis, "Observation of Ramsey Fringes Using a Stimulated, Resonance Raman Transition in a Sodium Atomic Beam," Phys. Rev. Lett., vol. 48, no. 13, pp. 867-870, Mar. 1982. [Online]. Available: http://prola.aps.org/abstract/PRL/v48/i13/p867_1

[4] J. Fuchs, G. J. Duffy, W. J. Rowlands, and A. M. Akulshin, "Electromagnetically induced transparency in ${ }^{6} \mathrm{Li}$," J. Phys. B, vol. 39, no. 17, pp. 3479-3489, 2006. [Online]. Available: http: //stacks.iop.org/0953-4075/39/3479

[5] S. Ulzega, "Theoretical and experimental study of the Stark effect in the ground state of alkali atoms in helium crystals," Ph.D. dissertation, Department of Physics, University of Fribourg, Switzerland, 2006, unpublished.

[6] S. Ulzega, A. Hofer, P. Moroshkin, and A. Weis, "Reconciliation of experimental and theoretical electric tensor polarizabilities of the cesium ground state," Europhys. Lett., vol. 76, no. 6, pp. 1074-1080, Dec. 2006, see errata in [14]. [Online]. Available: http://dx.doi.org/10.1209/epl/i2006-10383-2

[7] G. Di Domenico, L. Devenoges, A. Stefanov, A. Joyet, and P. Thomann, "Fourier analysis of Ramsey fringes observed in a continuous atomic fountain for in situ magnetometry," ArXiv e-prints, Apr. 2011.

[8] J. H. Shirley, "Velocity distributions calculated from the fourier transforms of ramsey lineshapes," IEEE Transactions on Instrumentation and Measurement, vol. 46, no. 2, pp. 117-121, Apr. 1997.

[9] J.-L. Robyr, P. Knowles, and A. Weis, "Stark shift of the cs clock transition frequency: a new experimental approach," Ultrasonics, Ferroelectrics and Frequency Control, IEEE Transactions on, vol. 57, no. 3, pp. 613-617, Mar. 2010.

[10] Z. Andjelic, POLOPT http://www.poloptsoftware.com/htm/modules portfolio.htm.

[11] P. Rosenbusch, S. Zhang, and A. Clairon, "Blackbody radiation shift in primary frequency standards," in Frequency Control Symposium, 2007 Joint with the 21st European Frequency and Time Forum. IEEE International, vol. 29, no. 1, June 2007, pp. 1060-1063.

[12] E. Simon, P. Laurent, and A. Clairon, "Measurement of the Stark shift of the Cs hyperfine splitting in an atomic fountain," Phys. Rev. A, vol. 57, no. 1, pp. 436-439, Jan. 1998. [Online]. Available: http://prola.aps.org/abstract/PRA/v57/i1/p436_1

[13] A. Godone, D. Calonico, F. Levi, S. Micalizio, and C. Calosso, "Starkshift measurement of the ${ }^{2} \mathrm{~S}_{1 / 2}, \mathrm{~F}=3 \rightarrow \mathrm{F}=4$ hyperfine transition of ${ }^{133}$ Cs," Phys. Rev. A, vol. 71, no. 6, p. 063401, 2005. [Online]. Available: http://link.aps.org/abstract/PRA/v71/e063401

[14] S. Ulzega, A. Hofer, P. Moroshkin, and A. Weis, "Erratum: Reconciliation of experimental and theoretical electric tensor polarizabilities of the cesium ground state," Europhys. Lett., vol. 78, no. 6, p. 69901, June 2007. [Online]. Available: http://dx.doi.org/10.1209/0295-5075/78/69901 